\title{
MODELING AND SIMULATION OF PE100 PIPE RESPONSE UNDER TRANSIENT EVENTS CAUSED BY PUMP FAILURE
}

\author{
Noura Bettaieb, Issa Chalghoum, Elaoud Sami, Ezzeddine Hadj Taieb \\ Research Laboratory "Applied Fluid Mechanics, Process Engineering and Environment" National Engineering School of \\ Sfax,Sfax,Tunisia; e-mail: noura.bettaieb@enis.tn; chalghoumissa@yahoo.fr; elaoudsa@yahoo.fr; e_hajtaieb@yahoo.fr
}

\begin{abstract}
In this work, the response of a PE100 pipe under transient events following pump failure is numerically investigated. The developed numerical model was based on the generalized Kelvin-Voigt model and the Vitkovsky et al. formulation. The method of characteristics (MOC) was used for numerical discretization. The relevance of an unsteady friction term in the pressure wave damping was analyzed. Pressure and circumferential stress responses indicated high rates in the pressure waves damping for the PE100 pipe. Through a parametric study, it was shown that the HDPE pipe may serve in damping and dispersing pressure waves without the need for additional protection devices.
\end{abstract}

Keywords: water hammer, PE100, pump failure, viscoelasticity, unsteady friction

\section{Introduction}

Water hammer or hydraulic transient are disturbances imposed on hydraulic networks upon a sudden interruption of the flow. Such disturbances may occur voluntarily during normal operations or in accidents and emergency cases (Chaudhry, 1979). The generated pressure waves usually follow fast valve operations or abrupt failure of pumping stations. The disruption in the flow conditions can cause pipeline failure, vibration, reduced system efficiency, and equipment damage. Accordingly, the analysis and control of this phenomenon are primordial due to the non-stopping growth of pumping stations along with the complexity of fluid transmission lines. Over the last 40 years, the ubiquitous usage of polymeric pipes in potable water distribution networks has escalated the attentiveness to the rheological behavior of pipe wall materials (Covas et al., 2004b). Polymeric pipes, in particular, High-Density Polyethylene (HDPE) pipes, are characterized by their mechanical behavior, simplicity of installation and low cost (Urbanowicz et al., 2016). The apprehension of the behavior of plastic pipe subjected to hydraulic disruptions is compulsory for better system performance. Subsequently, it is exceedingly vital to adopt water hammer solvers that assimilate supplementary effects, which are not consistently accessible on commercial software (e.g., pipe rheological behavior and unsteady friction).

Numerous studies have been conducted to study the response of polymeric pipes under transient events. Studies varied from experimental investigations to numerical simulations of the response of polymeric pipes (Covas et al., 2004a,b; Duan et al., 2010; Evangelista et al., 2015; Firkowski et al., 2019). To ensure an efficient numerical modeling, researchers have also investigated the relevance of some parameters in the numerical modeling of polymeric pipes. Under the frame of 1D modeling, it has been shown that viscoelasticity (VE) is preponderant compared to the impact of unsteady friction (UF) (Brunone and Berni, 2000; Brunone et al., 2004). In further studies, it was proven that the effects of UF and VE are commensurate only in the first stages of water hammer (Brunone and Berni, 2010; Duan et al., 2010). Specifically, it was found that viscoelasticity plays an increasingly vital role with the increase of time. Duan et al. (2012) conducted a dimensionless analysis to study the relevance of the unsteady friction 
term compared to the pipe system scale. It has been demonstrated that the impact of unsteady friction on transient pressure damping diminishes with the scale ratio of pipe length and pipe diameter.

Researchers have also considered the usage of polymeric pipe sections as transient control strategies. To address the limitations of conventional transient control devices, like surge tank relief valves or control valves, alternative control strategies were proposed. These techniques consist of implementing polymeric sections in the initial rigid networks. Previous studies have demonstrated the aptitude of such strategies on suppressing pressure surge in metallic networks. For instance, Ghilardi and Paoletti (1986) studied the transient response of a reservoir-pipeline-valve system with the integration of a polymeric section upstream the valve. They demonstrated that the additional polymeric section has the potential of reducing the initiated pressure surge following the closure of the valve. Hence, pressure wave oscillations were damped over time along the pipeline. However, it was also shown that an unduly short section could generate higher overpressures exciding those obtained in the unprotected network. Triki (2016) numerically studied the response of a simple reservoir-pipeline-valve system with the integration of a short inline polymeric section made of high-or low-density polyethylene (HDPE)/(LDPE). The control of positive and negative surge waves were both addressed in the study. The obtained results demonstrated the efficiency of the inline section in dispersing and damping pressure waves in the pipeline. Moreover, it was evinced that the sensitivity of the pressure peaks magnitude depended on the volume of the integrated polymeric section. In a further study, Triki (2018) reinvestigated the inline water hammer control strategy. It was reported that the inline technique amplified the radial strain peaks and caused the spread out of the wave oscillation period. Triki and Chaker (2019) proposed and investigated the aptitude of an innovative compound technique based inline strategy. The method employs HDPE-LDPE sub-short-sections combination attached to the main steel pipe. Results evinced that the proposed method mitigated excessive pressure variations. Moreover, it was shown that the compound technique presented an acceptable compromise between the attenuation of the pressure and the circumferential stress and resulted in a limitation of the spread of the oscillation period and the amplification of radial strain. However, most conducted studies are restricted to the simple reservoir-pipe-valve system, which is not very practical from an industrial point of view since most irrigation systems contain pumping stations with integrated control devices. Moreover, in spite of the demonstrated aptitude of the inline strategy based control techniques, it should be noted that they cannot be considered very reliable seeing that the investigated configurations and the cause of transients are narrow. Specifically, the scale of the considered hydraulic network was restricted to short pipes and small diameters. For instance, LDPE pipe is only available in small diameters, which is not the case in common practice.

The aim of the current research study is twofold: A transient solver that takes into consideration the viscoelasticity of the pipe wall material and the unsteady friction term is first developed. The efficiency of the model is validated with relevant experimental results from the literature. The second aim is to investigate the response of the PE100 when subjected to negative pressure waves caused by centrifugal pump failure. The relevance of the unsteady friction in the pressure damping term is also investigated. Damping and dispersing rates of pressure waves are discussed in further stages. The interaction between the pipe wall material and a flywheel attached to the pump motor shaft is also presented.

\section{Materials and methods}

Modeling of unsteady flows in closed conduits is based on the conventional momentum and continuity equations (Chaudhry, 1979). To account for the impact of pipe wall rheology, an additional term should be included in the continuity equation. 
- Continuity equation

$$
V A \frac{\partial \rho}{\partial p} \frac{\partial p}{\partial x}+A \frac{\partial \rho}{\partial p} \frac{\partial p}{\partial t}+\rho A \frac{\partial V}{\partial x}+\rho V \frac{\partial A}{\partial \varepsilon_{\varphi}} \frac{\partial \varepsilon_{\varphi}}{\partial x}+\rho \frac{\partial A}{\partial \varepsilon_{\varphi}} \frac{\partial \varepsilon_{\varphi}}{\partial t}=0
$$

where $\varepsilon_{\phi}$ is circumferential strain, $V$ is the average velocity of the flow, $p$ is pressure, $\rho$ is fluid density, $A$ is the cross section area of the pipe, $t$ is time, and $x$ is distance along the pipe. The circumferential strain can be expressed as follows

$$
d \varepsilon_{\phi}=\frac{d R}{R} \quad \frac{d A}{d \varepsilon_{\phi}}=2 A \quad \frac{d \rho}{\rho}=\frac{d p}{K}
$$

in which $R$ is the pipe radius and $K$ is the bulk modulus of elasticity of the fluid.

Taking into account the relationship between the pipe cross-section area and the circumferential strain (Eq. $(2.2)_{1}$ ), and implementing the state of fluid equation (Eq. (2.2)2), Eq. (2.1) can be expressed as follows

$$
\frac{\partial p}{\partial t}+V \frac{\partial p}{\partial x}+K \frac{\partial V}{\partial x}+2 K\left(\frac{\partial \varepsilon_{\phi}}{\partial t}+V \frac{\partial \varepsilon_{\phi}}{\partial x}\right)=0
$$

Since polyethylene pipes exhibit both viscous and elastic properties, they do not respond according to Hooke's law when subjected to certain instantaneous stress $\sigma_{0}$. In fact, the viscosity of the substance gives the polymeric material a time-dependent strain decomposed of instantaneous elastic response $\varepsilon_{e}^{\varphi}$ and a retarded viscous response $\varepsilon_{r}^{\varphi}$ as presented in Fig. 1.

(a)
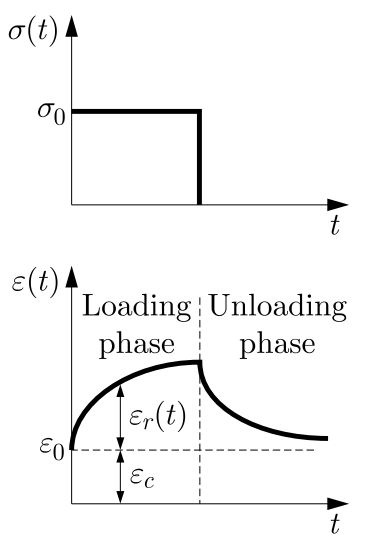

(b)
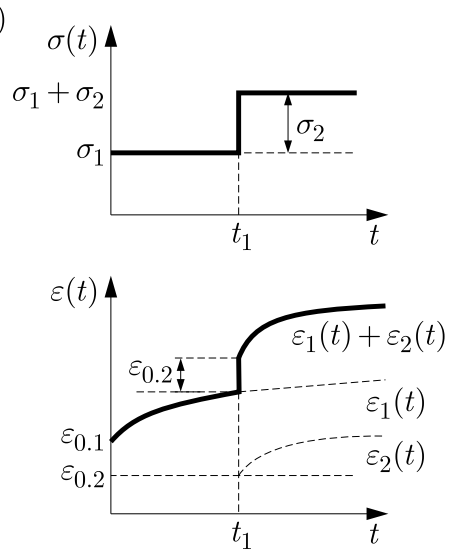

Fig. 1. (a) Stress for an instantaneous constant load, (b) Boltzmann superposition principle for two stresses applied sequentially (Covas et al., 2005)

Under these conditions, the expression of the total circumferential strain can be formulated as follows

$$
\varepsilon_{\varphi}=\varepsilon_{\varphi}^{e}+\varepsilon_{\varphi}^{r}
$$

If one assumes that the pipe material is linear viscoelastic, homogeneous and isotropic, and ignoring the inertia effect, the instantaneous elastic response $\varepsilon_{\varphi}^{e}$ can be expressed as

$$
\varepsilon_{\varphi}^{e}=\alpha\left(\frac{p R}{E_{0} e}\right)
$$

where $E_{0}$ is the Young modulus of elasticity, $D$ is the inner pipe diameter, $e$ is the thickness of the pipe wall and $\alpha$ is the pipe constraint factor. Consequently, the continuity equation can be formulated as

$$
\frac{\partial p}{\partial t}+V \frac{\partial p}{\partial x}+\rho a_{0}^{2} \frac{\partial V}{\partial x}+2 \rho a_{0}^{2}\left(\frac{\partial \varepsilon_{\phi}^{r}}{\partial t}+V \frac{\partial \varepsilon_{\phi}^{r}}{\partial x}\right)=0
$$


where the term $a_{0}$ represents the pressure wave celerity, expressed as

$$
a_{0}=\sqrt{\frac{1}{\rho}\left(\frac{1}{K}+\frac{2 \alpha R}{E_{0} e}\right)}
$$

The viscoelastic term of Eq. (2.6) can be modeled using the generalized Kelvin-Voigt (KV) model. Referring to Fig. 2, the model consists of a series association of $N_{k v}$ Kelvin-Voigt elements, solids of viscosity $\mu_{i}$ and creep compliance $J_{i}=1 / E_{i}$, and the compliance $J_{0}=1 / E_{0}$. This model is widely used for modeling the response of the pipe wall viscoelasticity during hydraulic transient events (Covas et al., 2005). Subsequently, the retarded strain can be expressed as follows

$$
\varepsilon_{\varphi}^{r}=\sum_{j=1}^{N_{k v}} \varepsilon_{\varphi}^{r, j}
$$

where $k$ is the number of $\mathrm{KV}$ elements.

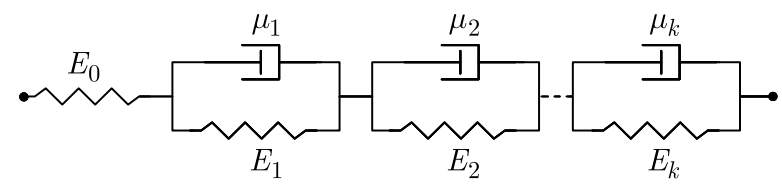

Fig. 2. Kelvin-Voigt model

The stress $\sigma$ can be formulated using the following expressions

$$
\sigma=E_{0} \varepsilon_{\varphi}^{e} \quad \sigma=E_{j}\left(\varepsilon_{\varphi}^{r ; j}+\tau_{j} \frac{\partial \varepsilon_{\varphi}^{r ; j}}{\partial t}\right) \quad j=1,2, \ldots, k
$$

where $\tau_{j}=\mu_{j} / E_{j}$ ( $\mu_{j}$ is the damping coefficient). Equalizing (2.8) and employing Eq. (2.5), the time derivative of the retarded strain can be expressed as follows

$$
\frac{\partial \varepsilon_{\varphi}^{r ; j}}{\partial t}=\frac{1}{\tau_{j}}\left(b_{j} p-\varepsilon_{\varphi}^{r ; j}\right) \quad j=1,2, \ldots, k
$$

where $b_{j}$ is given by $b_{j}=\alpha R /\left(E_{j} e\right)$.

- Momentum equation

The momentum equation can be written as

$$
\frac{\partial V}{\partial t}+V \frac{\partial V}{\partial x}+\frac{1}{\rho} \frac{\partial p}{\partial x}+h_{f}=0
$$

where $h_{f}$ represents the friction head losses in the system, which are calculated as the sum of steady and unsteady friction terms

$$
h_{f}=h_{f s}+h_{f u}
$$

The steady state term can be computed using the Darcy-Weisbach friction equation (Ghidaoui et al., 2005)

$$
h_{f s}=\frac{f V|V|}{2 D}
$$

where $D$ is the pipe diameter, $f$ is the friction coefficient which is estimated using the Colebrook formula given by: 
— for laminar flows

$$
f=\frac{64}{\operatorname{Re}}
$$

— for turbulent flows

$$
\frac{1}{\sqrt{f}}=-2 \log _{10}\left(\frac{k}{3.7 D}+\frac{2.51}{\operatorname{Re} \sqrt{f}}\right)
$$

in which $\operatorname{Re}=V D / v_{f}$ is the Reynolds number, $k$ is the pipe roughness and $v_{f}$ is the kinematic viscosity.

The unsteady friction term is computed using the Vitkovsky formulation (Vitkovsky et al., 2000). This unsteady friction formulation can be easily integrated into the MOC, without the need of complex axisymmetric models (Ramos et al., 2004)

$$
h_{f u}=\frac{k_{v}}{g}\left(\frac{\partial V}{\partial t}+a_{0} \operatorname{sgn}(V)\left|\frac{\partial V}{\partial x}\right|\right)
$$

where $k_{v}$ is the decay coefficient calculated by the formula proposed by Vardy and Brown (1995)

$$
k_{v}=2 \sqrt{\frac{7.41}{\operatorname{Re}}^{\log \left(14.3 / \mathrm{Re}^{0.05}\right)}}
$$

and

$$
\operatorname{sgn}(V)=\left\{\begin{array}{lll}
1 & \text { for } & V>0 \\
-1 & \text { for } & V<0
\end{array}\right.
$$

The fluid considered in this study is water. Hence, the fluid velocity can be considered negligible compared to the wave speed $V=a_{0}$. Accordingly, the convective terms in Eqs. (2.6) and (2.10) can be neglected.

\section{Numerical resolution method}

The previous set of partial differential equations can be numerically solved by various methods namely, finite elements, explicit or implicit finite differences, the method of characteristics (MOC) etc. In this study, the MOC with linear integration was used for the approximation of the linear governing equations. The MOC is often adopted for solving the 1D hydraulic transients in complex hydraulic systems (Chaudhry, 2014). This method is mainly characterized by its capability to illustrate the propagation of the wave through pipelines and to correctly simulate the steep wave fronts. Additionally, the simplicity of programming and efficiency of computations present major advantages of the method. The Courant-Friedrich-Lewy (CFL) stability condition is used to verify the stability of the finite-difference scheme. As described by the physics of the problem, if $C_{N}=C \Delta t / \Delta x=1$ the propagation of the wave is produced correctly. Therefore, the MOC is considered unconditionally stable since the computational time step is equal to $\Delta t=\Delta x / C$.

The momentum and continuity equations are transformed into a set of ordinary differential equations (3.1) valid along the characteristic lines $C^{ \pm}$presented in Fig. 3

$$
C^{ \pm}: \quad \frac{d H}{d t} \pm \frac{a_{0}}{g A} \frac{d Q}{d t}+\frac{2 a_{0}^{2}}{g}\left(\frac{\partial \varepsilon_{\varphi}^{r}}{\partial t}\right) \pm a_{0} h_{f}=0
$$




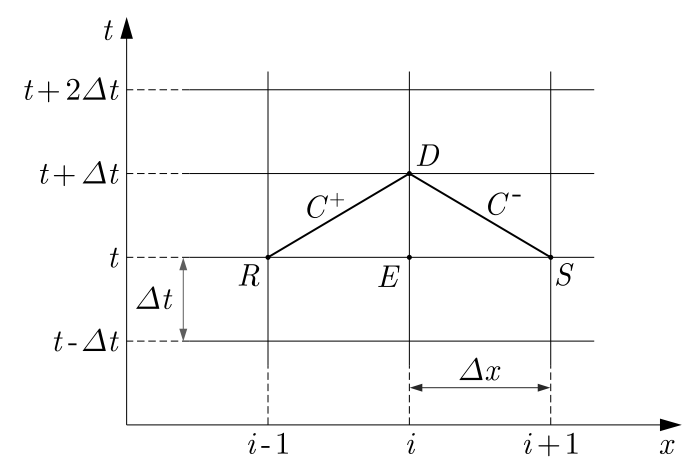

Fig. 3. Computational grid

The numerical scheme proposed by Soares et al. (2008) was used for calculating the convective and local terms of the unsteady friction

$$
\begin{aligned}
& \left.\frac{\partial Q}{\partial t}\right|_{C^{ \pm}}=\theta \frac{Q(x, t)-Q(x, t-\Delta t)}{\Delta t}+(1-\theta) \frac{Q(x \mp \Delta x, t-\Delta t)-Q(x \mp \Delta x, t-2 \Delta t)}{\Delta t} \\
& \left.\frac{\partial Q}{\partial x}\right|_{C^{ \pm}}=\frac{Q(x, t-\Delta t)-Q(x \mp \Delta x, t-\Delta t)}{\Delta x}
\end{aligned}
$$

and

$$
\left.\operatorname{sgn}(Q)\right|_{C^{ \pm}}=\operatorname{sgn}[Q(x \mp \Delta x, t-\Delta t)]
$$

where the parameter $\theta$ is a relaxation coefficient. In this study, this parameter was set to unity $\theta=1$ to minimize the computational effort. To compute the flow rate $Q$ and the head $H$ at the section $i$ of the pipe and instant $t$ of the time simulation, the following expressions are combined (Soares et al., 2013)

$$
\begin{aligned}
Q_{i, t} & =C_{P}-C_{a^{-}} H_{i, t} \\
Q_{i, t} & =C_{N}+C_{a^{+}} H_{i, t}
\end{aligned}
$$

where

$$
\begin{aligned}
& C_{P}=\frac{Q_{i-1, t-\Delta t}+\frac{g A}{a_{0}} H_{i-1, t-\Delta t}+C_{p 1}^{\prime}+C_{p 1}^{\prime \prime}+C_{p 1}^{\prime \prime \prime}}{1+C_{p 2}^{\prime}+C_{p 2}^{\prime \prime}} \\
& C_{N}=\frac{Q_{i+1, t-\Delta t}-\frac{g A}{a_{0}} H_{i+1, t-\Delta t}+C_{n 1}^{\prime}+C_{n 1}^{\prime \prime}+C_{n 1}^{\prime \prime \prime}}{1+C_{n 2}^{\prime}+C_{n 2}^{\prime \prime}} \\
& C_{a^{+}}=\frac{\frac{g A}{a_{0}}+C_{p 2}^{\prime \prime \prime}}{1+C_{p 2}^{\prime}+C_{p 2}^{\prime \prime}} \quad C_{a^{-}}=\frac{\frac{g A}{a_{0}}+C_{n 2}^{\prime \prime \prime}}{1+C_{n 2}^{\prime}+C_{n 2}^{\prime \prime}}
\end{aligned}
$$

Further coefficients are displayed as below:

- Steady state friction [']

$$
\begin{array}{lll}
C_{p 1}^{\prime}=-C_{0} \Delta t\left|Q_{i-1, t-\Delta t}\right| Q_{i-1, t-1} & C_{p 2}^{\prime}=0 & C_{0}=\frac{f}{2 D A} \\
C_{n 1}^{\prime}=-C_{0} \Delta t\left|Q_{i+1, t-\Delta t}\right| Q_{i+1, t-1} & C_{n 2}^{\prime}=0 &
\end{array}
$$


— Unsteady friction ["]

$$
\begin{aligned}
& C_{p 1}^{\prime \prime}=k_{v} \theta Q_{i, t-\Delta t}-k_{v}(1-\theta)\left(Q_{i-1, t-\Delta t}-Q_{i-1, t-2 \Delta t}\right) \\
& -k_{v} \operatorname{sgn}\left(Q_{i-1, t-\Delta t}\right)\left|Q_{i, t-\Delta t}-Q_{i-1, t-\Delta t}\right| \\
& C_{n 1}^{\prime \prime}=k_{v} \theta Q_{i, t-\Delta t}-k_{v}(1-\theta)\left(Q_{i+1, t-\Delta t}-Q_{i+1, t-2 \Delta t}\right) \\
& -k_{v} \operatorname{sgn}\left(Q_{i+1, t-\Delta t}\right)\left|Q_{i, t-\Delta t}-Q_{i+1, t-\Delta t}\right| \\
& C_{p 2}^{\prime \prime}=C_{n 2}^{\prime \prime}=k_{v} \theta
\end{aligned}
$$

— Rheological behavior of the pipe-wall material ["']

$$
\begin{aligned}
& C_{P 1}^{\prime \prime \prime}=-C_{N 1}^{\prime \prime \prime}=-2 a_{0} A \Delta t \sum_{k=1}^{N_{k v}}\left\{-\frac{b_{0} J_{k}}{\tau_{k}} H_{i, 0}+\frac{b_{0} J_{k}}{\tau_{k}}\left[1-\left(1-e^{-\frac{\Delta t}{\tau_{k}}}\right) \frac{\tau_{k}}{\Delta t}\right] H_{i, 0}\right. \\
& \left.-\frac{b_{0} J_{k}}{\tau_{k}}\left[\left(1-e^{-\frac{\Delta t}{\tau_{k}}}\right) \frac{\tau_{k}}{\Delta t}-e^{-\frac{\Delta t}{\tau_{k}}}\right]\left(H_{i, t-1}-H_{i, 0}\right)-\frac{e^{-\frac{\Delta t}{\tau_{k}}}}{\tau_{k}} \varepsilon_{r k(i, t-1)}\right\} \\
& C_{p 2}^{\prime \prime \prime}=C_{n 2}^{\prime \prime \prime}=2 a_{0} A \Delta t b_{0} \sum_{k=1}^{N_{k v}} \frac{J_{k}}{\Delta t}\left(1-e^{-\frac{\Delta t}{\tau_{k}}}\right)
\end{aligned}
$$

At $i=1$ and $i=N+1$, only one equation is valid along the characteristic lines. Thus, further equations are required to fully compute the head and discharge throughout the pipes. These equations are referred to as boundary conditions (BCs). In what follows, only the BC describing the pump transient state is presented.

\subsection{Viscoelastic approach validation}

To validate the previously developed model, experimental measurements provided by Covas et al. (2004b) were considered for numerical transient investigations. The investigated test facility consists of an upstream pressurized tank with volume $=750$ litre, which is connected to a downstream globe valve through a single Polyethylene pipe with a total length of $271.8 \mathrm{~m}$. The chosen pipe material was high-density polyethylene SDR11 PE100 NP16 with nominal diameter $N D=63 \mathrm{~mm}$, wall thickness $e=6.25 \mathrm{~mm}$ and internal diameter $D=50.6 \mathrm{~mm}$. The initial steady state discharge flow of the system was $Q_{0}=1.0 \mathrm{~s}^{-1}$. The flow disturbances in the reservoir-pipe system were initiated by fast closure of the downstream ball valve. A five-element KV model with creep coefficient and retardation time presented in Table 1 was used to model the linear viscoelastic behavior of the pipe wall.

Table 1. Calibrated creep function parameters (Covas et al., 2005)

\begin{tabular}{|l|c|c|c|c|c|c|}
\hline $\begin{array}{l}\text { Creep coeff. } \\
{\left[10^{-10} \mathrm{~Pa}\right]}\end{array}$ & $J_{0}=0.699$ & $J_{1}=1.057$ & $J_{2}=1.054$ & $J_{3}=0.9051$ & $J_{4}=0.2617$ & $J_{5}=0.7456$ \\
\hline $\begin{array}{l}\text { Retardation } \\
\text { time }[\mathrm{s}]\end{array}$ & - & $\tau_{1}=0.05$ & $\tau_{2}=0.5$ & $\tau_{3}=1.5$ & $\tau_{4}=5$ & $\tau_{5}=10$ \\
\hline
\end{tabular}

Numerical results of the head variations at the valve level (location T1) were compared with the experimental data from (Covas et al., 2004b). The obtained results with different numerical approaches are illustrated in Fig. 4. To account for the variations in the level of the pressurized tank generated by the traveling pressure wave, the upstream boundary condition was set as a variable level reservoir. The head at section $i=1$ was implemented in the transient solver as a function of time-based on the experimental data. As it can be observed from Fig. 4, the elastic water hammer model provides highly overestimated pressure amplitudes and phase compared to the experimental results. Meanwhile, the results of the viscoelastic transient solver with the 
Vitkovsky unsteady-friction formulations fit the experimental data exceedingly well. However, a slight positive shift is noticed in the subsequent cycles. This model is capable of reporting unsteady pressure wave damping, dispersion, and shape.

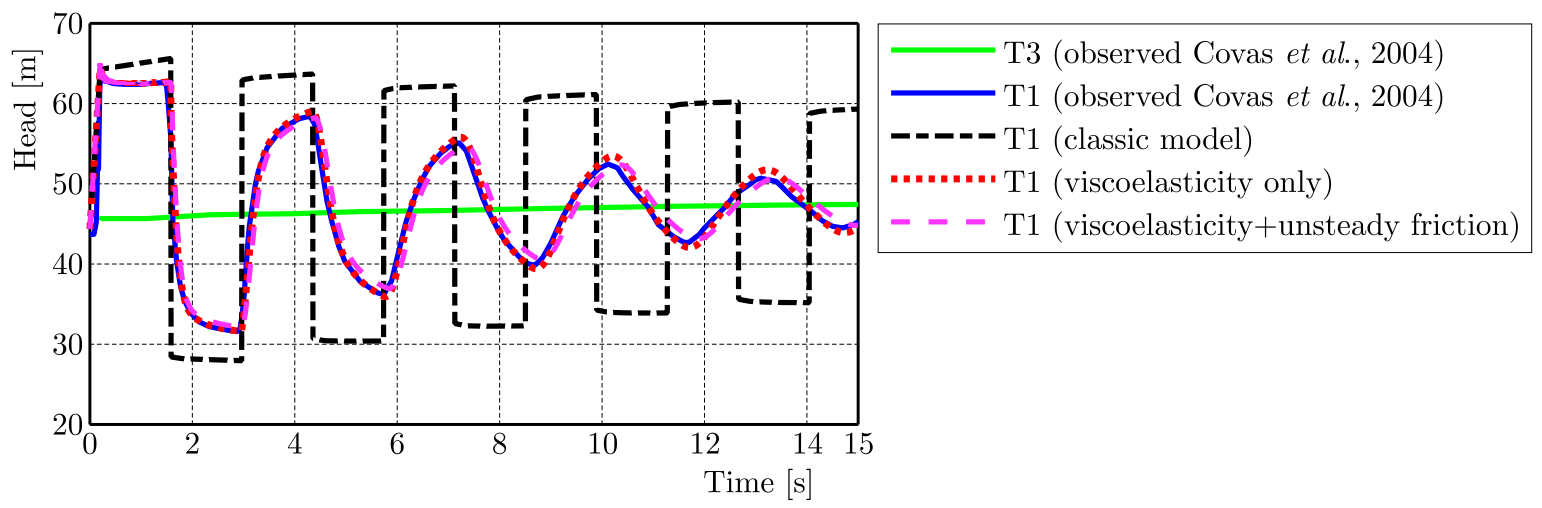

Fig. 4. Pressure variations at location T1 (distant $271.5 \mathrm{~m}$ from the upstream reservoir)

\section{Results and discussions}

\subsection{Response of a rigid steel pipe}

The response of a rigid steel pipeline subjected to a negative pressure wave was investigated for the network presented in (Frelin, 2002). The system presented in Fig. 5 consists of a centrifugal pump located at the upstream side, feeding a downstream reservoir through a rigid steel pipe. The characteristics of the installation are presented in Table 2. The flow disturbances in the hydraulic network were initiated by a sudden motor-pump disjunction. Following the pump trip, fluid reversal is likely to occur. Hence, a check valve is mounted on the discharge side of the pump to protect it from adverse effects. The numerical modeling of the unsteady flow in the pumping station was performed using the developed water hammer model.

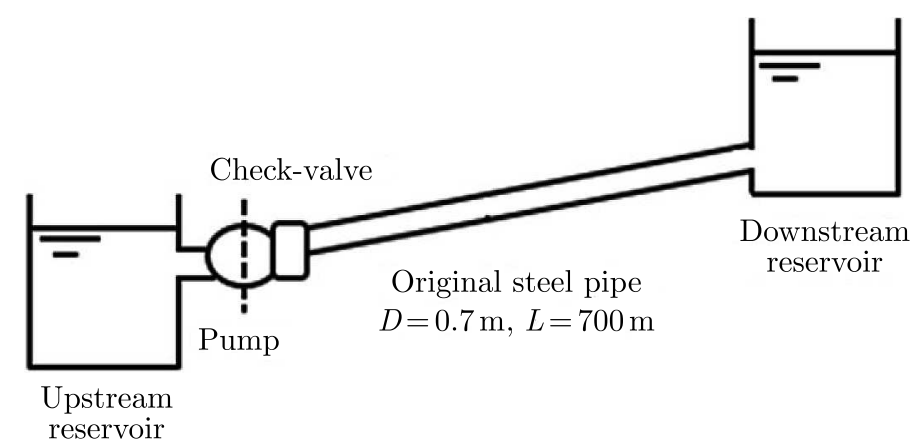

Fig. 5. Water supply installation

Table 2. Characteristics of the hydraulic installation

\begin{tabular}{|c|c|c|c|c|}
\hline Parameter & Pumping head & Flow rate & Total inertia & Rotational speed \\
\hline \hline Value & $32 \mathrm{~m}$ & $0.3 \mathrm{~m}^{3} / \mathrm{s}$ & $20 \mathrm{~kg} \mathrm{~m}^{2}$ & $1500 \mathrm{rpm}$ \\
\hline
\end{tabular}

Equations describing the hydraulic performance of the pump following the sudden disjunction are used as an upstream boundary condition. For the downstream boundary, the reservoir was assumed to maintain a constant head during the transient events. In common practice, the water 
level changes over time during transient events (Soares et al., 2013). However, for simplification reasons, the water level was considered constant during the simulation.

Formulations describing the pump failure boundary condition are presented in (Chaudhry, 2014). The formulations are based on the dimensional similarity principle. Marchal et al. (1965) provided the complete characteristic curves of pumps, which provide two curves used to describe the performance of the pump at different values of the rotational speed. The fitting curves strongly depend on other known complete performance curves of geometrically similar pumps. Such a used method may bring some error in transient simulation (Wan and Huang, 2011). Therefore, a method based on the experimental performance curves of the pump would yield more accurate results.

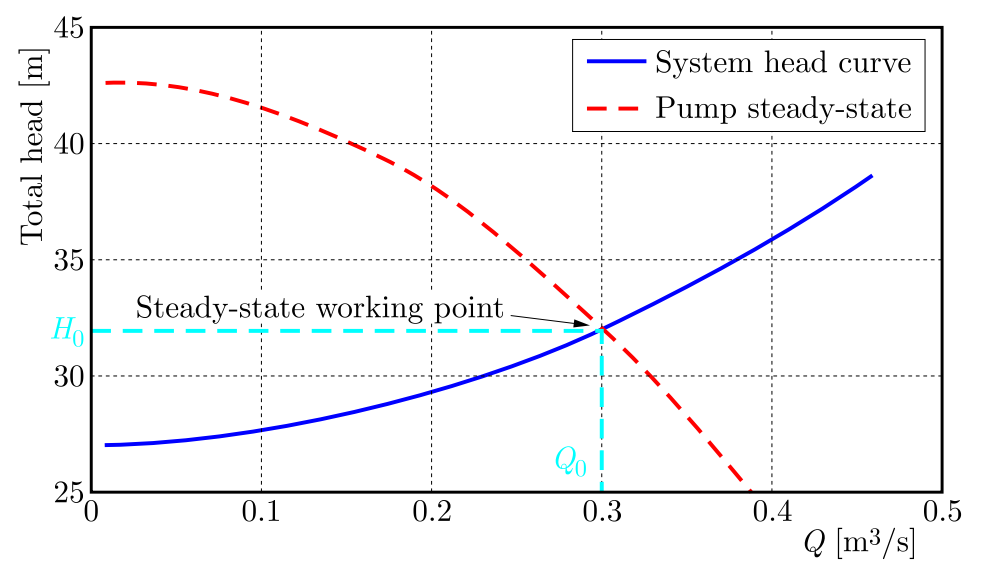

Fig. 6. Head discharge curve at $N=1500 \mathrm{rpm}$ and the system head curve

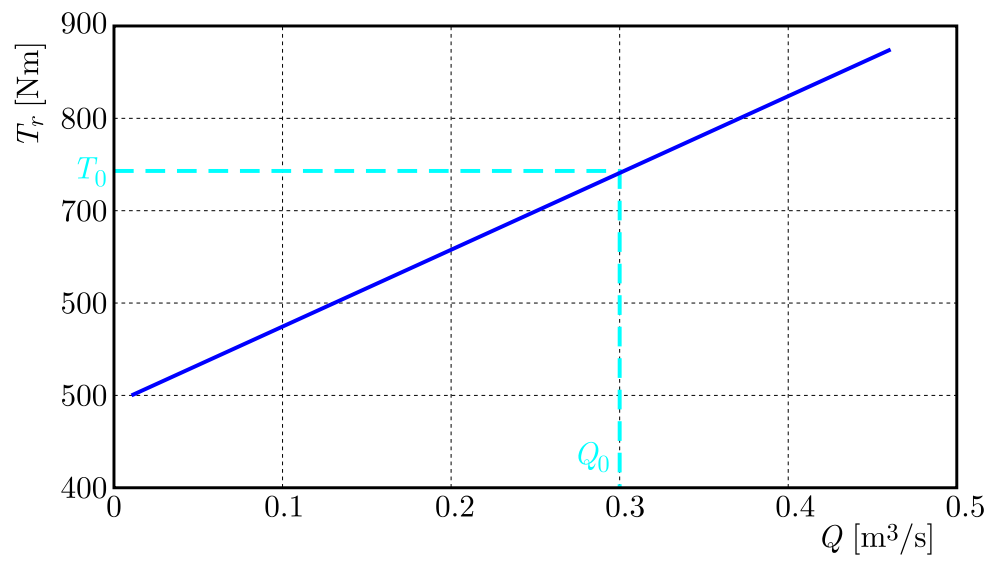

Fig. 7. Resistant torque of the pump $V_{s}$ discharge at $N=1500 \mathrm{rpm}$

The head characteristic curve of the pump plotted in Fig. 6 can be approximated by a second-order polynomial function, Eq. (4.1), while the resistant torque plotted in Fig. 7 can be approximated by a linear function, Eq. (4.2)

$$
H=a \omega^{2}-b_{0} Q^{2}
$$

in which $\omega$ is the rotational speed. For $N=1500 a \omega_{i}^{2}=42 \mathrm{~m}$ and $b_{0}=120 \mathrm{~s}^{2} / \mathrm{m}^{5}$

$$
T_{\text {resistant }}=a_{1} \omega^{2}+a_{2} \omega Q
$$

in which $a_{1}=0.02 \mathrm{~kg} \mathrm{~m}^{2}$ and $a_{2}=5.236 \mathrm{~kg} / \mathrm{m}$. 
If we assume that $t_{0}=0 \mathrm{~s}$ corresponds to the instant of motor-pump disjunction, the rotational speed would be equal to $N=N_{0}$, the discharge flow rate $Q=Q_{0}$, pumping head $H=H_{0}$ and torque $T=T_{0}=740 \mathrm{Nm}$. The time-varying rotational speed of the pump can be computed using the axial torque equation written as follows

$$
I \frac{d \omega}{d t}=T_{\text {motor }}-T_{\text {resistant }}
$$

where $T_{\text {motor }}$ is the motor torque, $T_{\text {resistant }}$ is the resistant torque and $I$ is the sum of the inertia of the rotating masses around the rotating axis, the fluid, and the flywheel connected to the shaft of the pump. At any rotational speed, the resistant torque of the pump is known. At the disjunction of the pump from its motor, the motor torque is switched off which yields the following expression

$$
I \frac{d \omega}{d t}=-T_{\text {resistant }}
$$

Several methods have been introduced in the literature to integrate this equation. These methods consist on representing the resistant torque as a function of the speed and the discharge. Integrating Eq. (4.4) between the initial speed $\omega_{i}$ and the final speed $\omega_{f}$ and replacing the expression of $T_{\text {resistant }}$ with Eq. (4.2) yield the following expression

$$
\int_{\omega_{i}}^{\omega_{f}} \frac{1}{a_{1} \omega^{2}+a_{2} \omega Q_{i}} d \omega=-\frac{1}{I} \int_{t_{i}}^{t_{f}} d t
$$

where $\omega_{i}$ is obtained from the previous time step $t=t_{i}$. Assuming that the flow rate $Q_{i}$ remains constant during the time step $\Delta t=\left(t_{f}-t_{i}\right)$, the rotational speed $\omega_{f}$ can be approximated by Eq. (4.6)

$$
\omega_{f}=\frac{a_{2} Q_{i}}{\left(a_{1}+\frac{a_{2} Q_{i}}{\omega_{i}}\right) \exp \left(\frac{a_{2} Q_{i}}{I}\left(t_{f}-t_{i}\right)-a_{1}\right)}
$$

At section $i=1$, compatibility Eq. (3.4) along the negative characteristic line $C^{-}$are used to compute the pump head as follows

$$
H_{P 1}=\frac{Q_{P 1}-C_{n}}{C_{a^{-}}}
$$

Hence, the discharge of the pump is computed by combining both Eq. (4.7) and Eq. (4.1) and replacing the rotational speed with Eq. (4.6)

$$
Q_{P 1}=\frac{1}{2 b_{0} C_{a^{-}}}\left(-1+\sqrt{1+4 b_{0} C_{a^{-}}\left(a_{0} C_{a^{-}} \omega_{f}^{2}+C_{N}\right)}\right)
$$

The head of the pump $H_{P 1}$ during the transient events is obtained using Eq. (4.7). The outlined pump failure formulations are capable to simulate pump failure events without the flow or speed reversal. To validate the applicability of the developed pump boundary condition, the numerically obtained results were compared with those presented in literature (Frelin, 2002). Figure 8 confirms that the pump boundary condition yields reliable results. A good agreement was obtained between the results obtained using the MOC and those obtained using the Graphical method of Bergeron.

Following the abrupt motor-pump disconnection, a negative pressure wave is initiated downstream the pump and accompanied with a decrease in the total head reaching a maximum value 


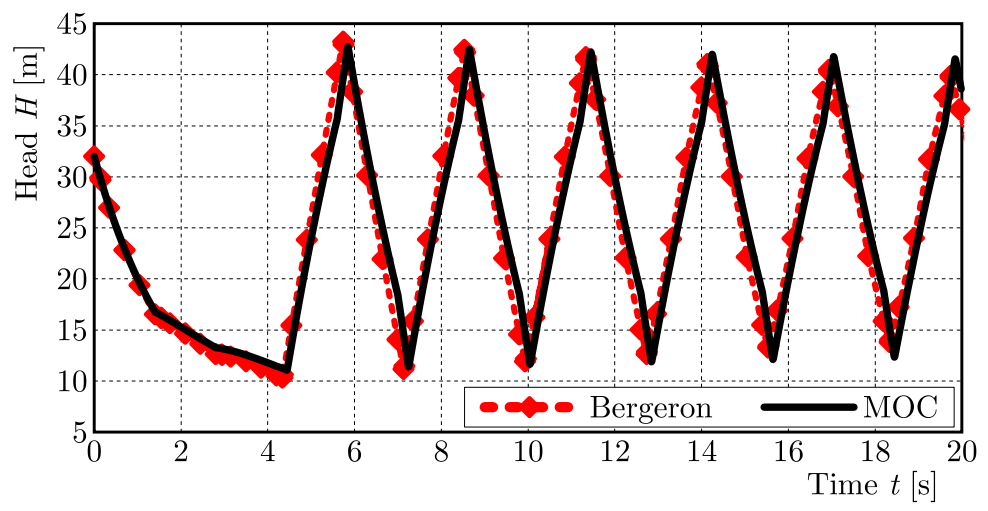

Fig. 8. Head fluctuation downstream the pump

of $\Delta H=20.92 \mathrm{~m}$ at time instant $t=4.45 \mathrm{~s}$. The first generated negative pressure wave travels from the pump through the discharge line until it reaches the downstream reservoir. As indicated in Fig. 9, the pressure wave causes a drop in the pressure at different sections of the pipe. When reaching the downstream reservoir at $t=L / C=0.7 \mathrm{~s}$, the wave is reflected and returns as a positive pressure wave causing the pressure to rise along the pipe. As the flow returns from the reverse direction, the drag of the fluid closes the check valve, which causes an abrupt rise in the pressure.

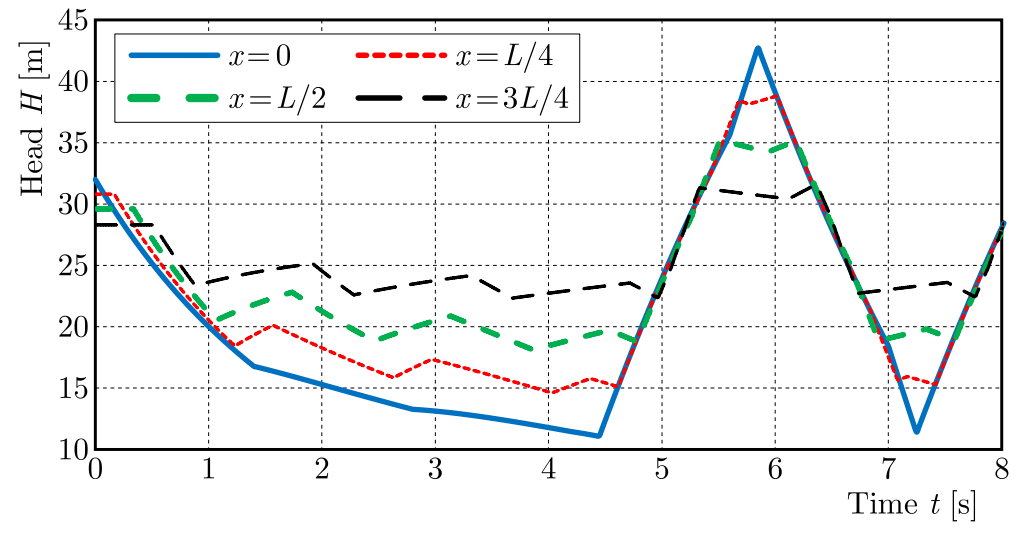

Fig. 9. Head fluctuations at different locations

\subsection{Response of PE100 subjected to negative pressure waves}

As outlined previously, HDPE pipes are highly capable of damping and dispersing pressure waves. To investigate the response of a polymeric pipe subjected to a negative pressure wave, the rigid steel pipe in the previous pumping station was replaced with a high-density polyethylene pipe. The characteristics of the considered PE100 pipe are illustrated in Table 3.

Table 3. Characteristics of the PE100 pipe

\begin{tabular}{|c|c|c|c|c|c|}
\hline Parameter & $\begin{array}{c}\text { Inner } \\
\text { diameter }\end{array}$ & $\begin{array}{c}\text { Outer } \\
\text { diameter }\end{array}$ & Thickness & $\begin{array}{c}\text { Wave } \\
\text { speed }\end{array}$ & $\begin{array}{c}\text { Normal service } \\
\text { pressure }\end{array}$ \\
\hline \hline HDPE & $0.705 \mathrm{~m}$ & $0.8 \mathrm{~m}$ & $0.047 \mathrm{~m}$ & $313.5 \mathrm{~m} / \mathrm{s}$ & $10 \mathrm{bar}$ \\
\hline
\end{tabular}

Figure 10 indicates that pressure waves obtained by the viscoelastic model, with and without the unsteady friction term, are damped and dispersed over time, which is attributed to the low rigidity of PE100. Obtained results are physically well interpreted considering that HDPE pipes 
result in a predominant pressure wave diminishment. A closer look to Fig. 10 reveals that both assumptions exhibit a comparable pressure head attenuation in the first stage of the flow perturbations. In further stages, the unsteady friction term engenders a slight positive shift in the phase.

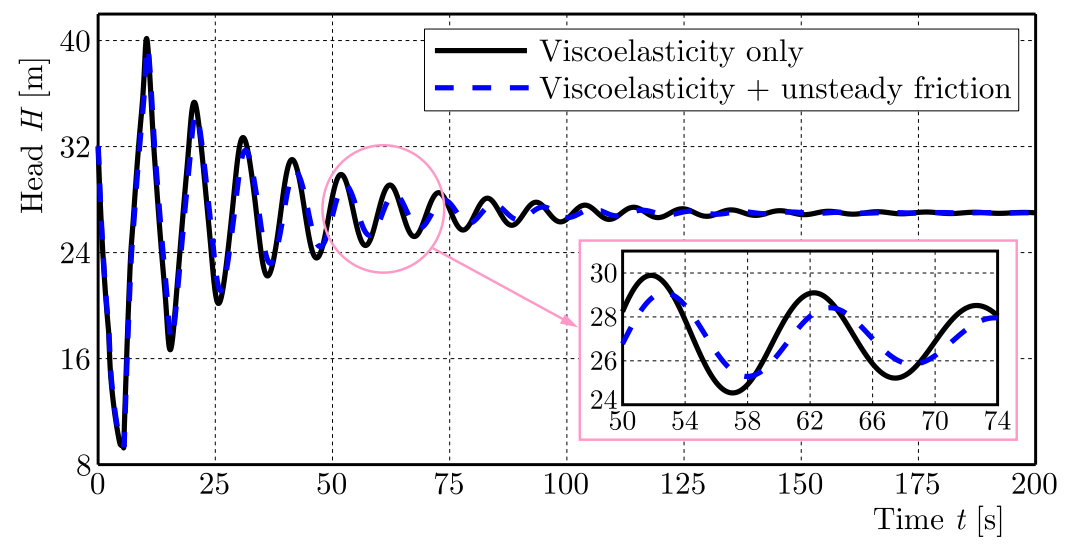

Fig. 10. Head downstream the pump following the motor-pump disjunction

The relevance of the unsteady friction contribution in pressure damping can be further verified using a dimensional parameter $I_{d}$ provided in (Duan et al., 2012). The formulations are defined as follows

$$
T_{w}=\frac{L}{a} \quad T_{d}=\frac{D^{2}}{v_{k}} \quad I_{d}=\frac{f \operatorname{Re} T_{w}}{T_{d}}=\frac{f M L}{D}
$$

where Re is the Reynolds number and $M=V / a$ is the Mach number. Duan et al. (2012) stated that the relevance of unsteady friction decreases as $f$ Re and $T_{w} / T_{d}$, or correspondingly, when $f M$ and $L / D$ increase. Consequently, the contribution of unsteady friction diminishes with the increase of the hydraulic system scale, equivalently with the increase of $I_{d}$. In their work, Duan et al. (2012) presented an analytical approximation, based on curve fitting, of the unsteady friction relevance $\gamma_{\text {fitted }}$ to the total damping. The dimensional parameter obtained for the present network is $I_{d}=0.0486$. Based on this value, Eq. (4.10) is used to approximate the percentage of the unsteady friction contribution in pressure damping

$$
\gamma_{\text {fitted }}=5.1 \cdot 10^{-2}\left(I_{d}\right)^{-0.31} \quad \text { for } \quad 10^{-4} \leqslant I_{d}<10^{-2}
$$

Since $\gamma_{\text {fitted }}=13.02 \%$, the contribution of the unsteady friction in pressure damping can be considered relatively low. However, in further calculations, the unsteady friction term is considered.

\subsection{Stress evaluation}

As outlined previously, rigid steel pipe failed dramatically in damping pressure waves. Moreover, the pipeline is subjected to cyclic loading with a frequency $f=0.357 \mathrm{~Hz}$. Hence, fatigue failure related to long duration cyclic pressure variation is likely to occur. Henceforth, an evaluation of the circumferential stress would be of major interest.

The normal circumferential-stress evaluated for a thin pipe-wall subjected to internal pressure $p$ can be expressed as follows

$$
\sigma=\frac{\alpha \Delta p R}{e}
$$

Figure 11 shows that the magnitude of the first circumferential-stress ridge is equal to $\Delta \sigma_{\text {steel }}=0.8 \mathrm{MPa}$. Likewise, a lower value is observed for the PE100 pipe $\Delta \sigma_{\mathrm{PE} 100}=0.5 \mathrm{MPa}$, 
which is attributed to the low rigidity of the polymeric pipe. A further observation from Fig. 11 illustrates that the stress variations imposed on the pipe wall stabilize in approximately $t=100 \mathrm{~s}$ for the PE100 pipe, whereas the steel pipe suffers from a continuous stress variation over time.

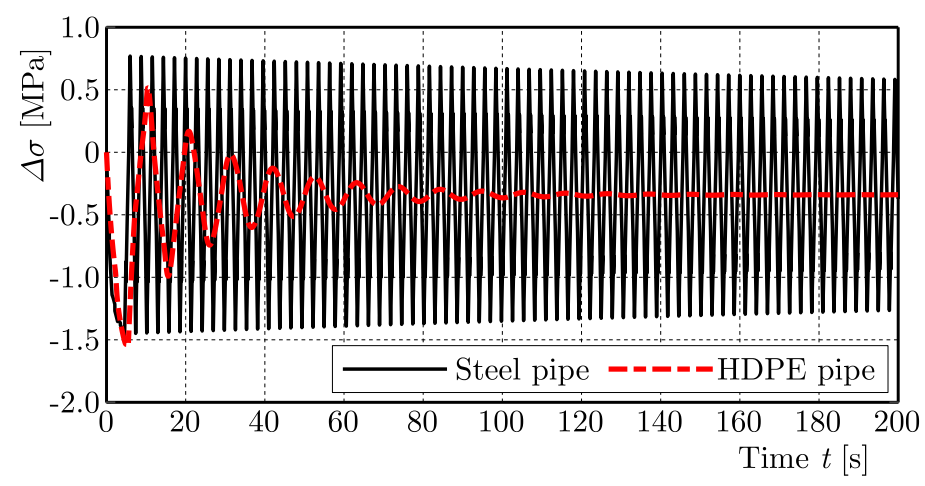

Fig. 11. Comparison of the circumferential stress evolution for two different materials

\subsection{Flywheel effect evaluation}

As mentioned previously, the pump motor shaft is equipped with a flywheel to control hydraulic transient. This device engenders a longer pump shutdown time, which means a continuous movement of the fluid (Wan et al., 2019). However, it should be noted that larger rotating masses require additional starting energy. Henceforth, a parametric study should be conducted to investigate the relevance of increasing the moment of inertia (MOI) in relation to the pipe wall material. The numerical analysis was conducted to study the pressure variations in both materials under different values of MOI. The value of MOI varied between a minimum $I=2 \mathrm{~kg} \mathrm{~m}{ }^{2}$ and a maximum $I=20 \mathrm{~kg} \mathrm{~m}^{2}$.
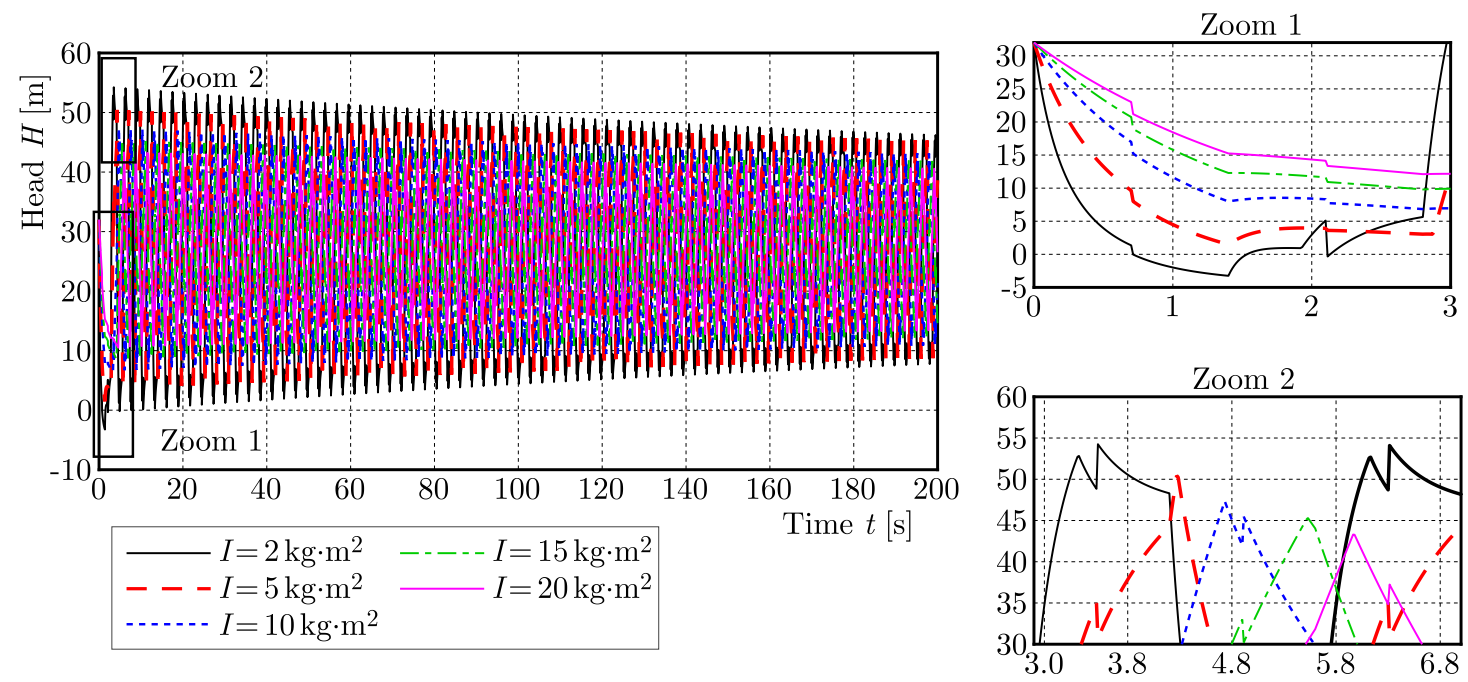

Fig. 12. Pressure variation under different moment of inertia: steel pipe

Figures 12 and 13 indicate an impulse wave pattern of the flow behavior with different MOI for steel and PE100, respectively. Zoom 1 indicates the pressure drop immediately following the pump failure, whereas Zoom 2 presents the highest reached pressure head. As expected, the pressure response in the steel pipe reached the maximum and minimum values. Specifically, under the lowest value of MOI, the pressure head dropped to reach the minimum value $\min H_{\text {steel pipe }}^{I=2 \mathrm{~kg} \mathrm{~m}^{2}}=-3.35 \mathrm{~m}$ and the maximum value $\max H_{\text {steel pipe }}^{I=2 \mathrm{~kg} \mathrm{~m}^{2}}=54.25 \mathrm{~m}$. Subsequently, as 
illustrated in Table 4, larger MOI values induce approximately a head difference $\Delta H=2 \mathrm{~m}$ between two subsequent MOI values. As for the PE100 pipe, the obtained values highlight that as far as the MOI increases, the values of the minimum and maximum ridges of the transient head present a relatively slight difference. For instance, the minimum and maximum reached head presented comparable values for $I=2 \mathrm{~kg} \mathrm{~m}^{2}$ and $I=5 \mathrm{~kg} \mathrm{~m}^{2}$. The differences in the minimum and maximum ridges for further MOIs are comparable to the case of the steel pipe. Nevertheless, as shown in Fig. 13, pressure variations are damped in all cases. On the contrary, as illustrated in Fig. 12, pressure exhibits continuous strong variations along time for different MOIs.
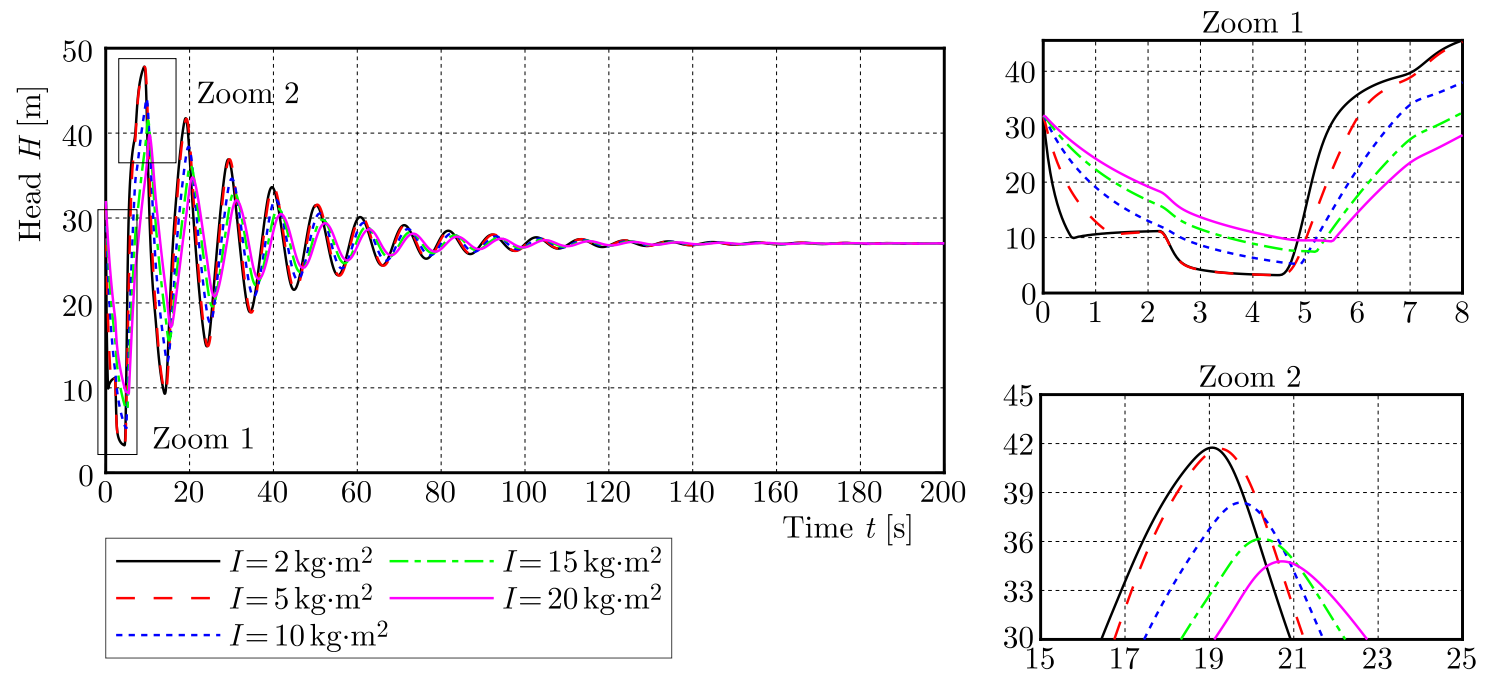

Fig. 13. Pressure variation under different moments of inertia: PE100 pipe

Table 4. Minimum and maximum pressure values for different values of MOI

\begin{tabular}{|l|c|c|c|c|c|c|c|c|c|c|}
\hline \multirow{2}{*}{ Parameter } & \multicolumn{2}{|c|}{$I=2 \mathrm{~kg} \mathrm{~m}^{2}$} & \multicolumn{2}{c|}{$I=5 \mathrm{~kg} \mathrm{~m}^{2}$} & \multicolumn{2}{c|}{$I=10 \mathrm{~kg} \mathrm{~m}^{2}$} & \multicolumn{2}{c|}{$I=15 \mathrm{~kg} \mathrm{~m}^{2}$} & \multicolumn{2}{c|}{$I=20 \mathrm{~kg} \mathrm{~m}^{2}$} \\
\cline { 2 - 11 } & Steel & PE100 & Steel & PE100 & Steel & PE100 & Steel & PE100 & Steel & PE100 \\
\hline \hline Min. head & -3.35 & 3.229 & 1.483 & 3.216 & 6.637 & 5.189 & 8.622 & 7.441 & 10.5 & 9.318 \\
\hline Max. head & 54.25 & 47.83 & 50.44 & 47.80 & 47.32 & 44.08 & 45.31 & 41.54 & 42.73 & 39.79 \\
\hline
\end{tabular}

\section{Conclusion}

In this study, a hydraulic transient analysis was performed to study the performance of the PE100 pipe when subjected to negative pressure waves. The analysis was conducted by a viscoelastic water hammer model which was validated with experimental results from the literature. The method of characteristics (MOC) with linear integration was used to solve the system of partial differential equations. A boundary condition, based on the pump experimental data, was adopted to study the pump trip events. Through a dimensionless analysis, it was shown that the role of the unsteady friction term in pressure damping was relatively small for the considered pump station. A parametric study was also performed to study the impact of using different sizes of flywheels as transient control devices in relation to the pipe wall material. Based on the numerically obtained results, the following conclusions are drawn:

In contrast to the rigid steel pipe, the viscoelastic property of the PE100 presented high capacity in attenuating pressure fluctuations throughout the pipeline. Sudden pressure variations were diminished over time which highly outline that it is vital to account for the rheological behavior of the material during transient simulation. HDPE pipes may serve in damping and dispersing pressure waves without the need of additional protection devices. 


\section{References}

1. Brunone B., Berni A., 2010, Wall shear stress in transient turbulent pipe flow by local velocity measurement, Journal of Hydraulic Engineering, 136, 10, 716-726, DOI: 10.1061/(ASCE)HY.19437900.0000234

2. Brunone B., Ferrante M., Cacciamani M., 2004, Decay of pressure and energy dissipation in laminar transient flow, Journal of Fluids Engineering, 126, 6, 928

3. Brunone B., Karney B.W., Mecarelli M., Ferrante M., 2000, Velocity profiles and unsteady pipe friction in transient flow, Journal of Water Resources Planning and Management, 128, $1,236-244$

4. Chaudhry M.H., 1979, Applied Hydraulic Transients, Van Nostrand Reinhold Company, New York

5. Chaudhry M.H., 2014, Applied Hydraulic Transients, Springer, New York, Heidelberg, Dordrecht, London

6. Covas D., Stoianov I., Mano J.F., Ramos H., Graham N., Maksimovic C., 2005, The dynamic effect of pipe-wall viscoelasticity in hydraulic transients. Part II - Model development, calibration and verification, Journal of Hydraulic Research, 43, 1, 56-70, DOI: 10.1080/00221680509500111

7. Covas D., Stoianov I., Ramos H., Graham N., Maksimovic C., 2004a, The dynamic effect of pipe-wall viscoelasticity in hydraulic transients. Part I - Experimental analysis and creep characterization, Journal of Hydraulic Research, 42, 5, 517-532

8. Covas D., Stoianov I., Ramos H., Graham N., Maksimović Č., Butler D., 2004b, Water hammer in pressurized polyethylene pipes: conceptual model and experimental analysis, Urban Water Journal, 1, 2, 177-197

9. Duan H.F., Ghidaoui M., Lee P.J., Tung Y.K., 2010, Unsteady friction and visco-elasticity in pipe fluid transients, Journal of Hydraulic Research, 48, 3, 354-362

10. Duan H.F., Ghidaoui M.S., Lee P.J., Tung Y.K., 2012, Relevance of unsteady friction to pipe size and length in pipe fluid transients, Journal of Hydraulic Engineering, 138, 2, 154-166

11. Evangelista S., Leopardi A., Pignatelli R., De Marinis G., 2015, Hydraulic transients in viscoelastic branched pipelines, Journal of Hydraulic Engineering, 141, 8, 04015016

12. Firkowski M., Urbanowicz K., Duan H.F., 2019, Simulation of unsteady flow in viscoelastic pipes, SN Applied Sciences, 1, 6, 519, DOI: 10.1007/s42452-019-0524-2

13. Frelin M., 2002, Coups de bélier, Techniques de l'ingénieur, 1-27

14. Ghidaoui M.S., Zhao M., McInnis D.A., Axworthy D.H., 2005, A review of water hammer theory and practice, Applied Mechanics Reviews, 58, 1, 49

15. Ghilardi P., Paoletti A., 1986, Additional viscoelastic pipes as pressure surges suppressors, Proceedings of 5th International Conference on Pressure Surges, BHR Groups, Cranfield, U.K.

16. Marchal M., Flesh G., Suter, P., 1965, The calculation of waterhammer problems by means of the digital computer, Proceedings of International Symposium Waterhammer Pumped Storage

17. Ramos H., Covas D., Borga A., Loureiro D., 2004, Surge damping analysis in pipe systems: modelling and experiments, Journal of Hydraulic Research, 42, 4, 413-425

18. Soares A., Covas D.I., Ramos H.M., 2013, Damping analysis of hydraulic transients in pump-rising main systems, ASCE Journal of Hydraulic Engineering, 139, 1, 233-243

19. Soares A.K., Covas D.I., Reis L.F., 2008, Analysis of PVC pipe-wall viscoelasticity during water-hammer, Journal of Hydraulic Engineering, 134, 9, 1389-1394

20. TRIKI A., 2016, Water-hammer control in pressurized-pipe flow using an in-line polymeric short-section, Acta Mechanica, 227, 3, 777-793 
21. Triki A., 2018, Further investigation on water-hammer control inline strategy in water-supply systems, Journal of Water Supply: Research and Technology - AQUA, 67, 1, 30-43

22. Triki A., Chaker M.A., 2019, Compound technique - based inline design strategy for water-hammer control in steel pressurized-piping systems, International Journal of Pressure Vessels and Piping, 169, 188-203, DOI: 10.1016/j.ijpvp.2018.12.001

23. Urbanowicz K., Firkowski M., Zarzycki Z., 2016, Modelling water hammer in viscoelastic pipelines: short brief, Journal of Physics: Conference Series, 760, 1, 012037

24. Vardy A.E., Brown J.M.B., 1995, Transient, turbulent, smooth pipe friction, Journal of Hydraulic Research, 33, 4, 435-456

25. Vitkovsky J.P., Lambert M.F., Simpson A.P., 2000, Advances in unsteady friction modelling in transient pipe flow, Proceedings of 8th International Conference on Pressure Surges-Safe Design and Operation of Industrial Pipe systems, 471-498

26. WAN W., HUANG W., 2011, Investigation on complete characteristics and hydraulic transient of centrifugal pump, Journal of Mechanical Science and Technology, 25, 10, 2583-2590

27. WAN W., Zhang B., Chen X., 2019, Investigation on water hammer control of centrifugal pumps in water supply pipeline systems, Energies, 12, 1

Manuscript received January 31, 2019; accepted for print June 14, 2019 